\title{
Relatividad y el Sistema de Posicionamiento Global (GPS)
}

\author{
Fulgencio Villegas ${ }^{* 1}$ \\ ${ }^{1}$ Universidad Nacional Mayor de San Marcos, Facultad de Ciencias Físicas, Lima, Perú
}

Recibido 25 enero 2020 - Aceptado 03 marzo 2020

\begin{abstract}
Resumen
En este artículo se explica los efectos relativistas en el Sistema de Posicionamiento Global (GPS); se analiza las correcciones debido a la Relatividad Especial y a la Relatividad General. Aunque los efectos gravitacionales son débiles y las velocidades pequeñas, sin embargo generan efectos relativistas significativos. Si dichos efectos no se tienen en cuenta, se producirían errores en la medicion del tiempo y en la posición del dispositivo GPS (en el orden de los $11.5 \mathrm{~km}$ ).
\end{abstract}

Palabras clave: Relatividad Especial, Relatividad General, Sistema de Posicionamiento Global.

\section{Relativity and the Global Positioning System (GPS)}

\begin{abstract}
In this article we explain the relativistic effects in the Global Positioning System (GPS); Corrections due to Special Relativity and General Relativity are analyzed. Although the gravitational effects are weak and the speeds are small, they nevertheless generate significant relativistic effects. If these effects are not taken into account, errors would occur in the measurement of time and in the position of the GPS device (in the order of $11.5 \mathrm{~km}$ ).
\end{abstract}

Keywords: Especial Relativity, General Relativity, Global Positioning System.

\section{Introducción}

Actualmente la tecnología satelital permite determinar, con muy buena precisión hasta del orden de metros, la posición espacial de objetos fijos o móviles sobre la superficie terrestre medidos respecto de un sistema de referencia fijo a la Tierra.

El Sistema de Posicionamiento Global (GPS) fue desarrollado por el Departamento de Defensa de los Estados Unidos para proporcionar un sistema de navegación por satélite para el ejército de los Estados Unidos. Más tarde se puso bajo control conjunto del Departamento de Defensa y el Departamento de Transporte para proporcionar usos de navegación tanto militares como civiles [Elr02].

El GPS en la actualidad se ha convertido en parte de la vida diaria y es utilizado prácticamente en todas las actividades de la vida humana: es utilizado en la industria de la telefonía móvil, es usado en los vehiculos mayormente para seguir su posición y movimiento, es útil a la policía para realizar seguimiento, tiene vital importancia en la navegación marítima, útil para obtener información

\footnotetext{
*fvillegass@unmsm.edu.pe
}

de la topografía marina y colocación de boyas, tambien es útil en la navegación aérea pues permite a los aviones volar con una ruta precisa.

La configuración del GPS consta de una red no menor de 24 satélites, que se distribuyen en 6 planos orbitales con 4 satélites cada uno, alrededor de la Tierra a una altitud de aproximadamente $20,000 \mathrm{~km}$ del suelo. Las órbitas de los satélites se distribuyen de manera que al menos 4 satélites sean siempre visibles desde cualquier punto de la Tierra en cualquier instante dado. Cada satélite lleva consigo un reloj atómico que funciona con una precisión de 1 nano segundo.

Técnicas sofisticadas, como los métodos de GPS diferencial y cinemática en tiempo real, permiten determinar posiciones con un alto grado de precisión empleando poco tiempo de medición. Para lograr este nivel de precisión, los tics de reloj de los satélites GPS deben conocerse con una precisión de 20 a 30 nanosegundos. Sin embargo, debido a que los satélites se mueven constantemente en relación con los observadores en la Tierra, los efectos predichos por las Teorías de Relatividad Especial y General deben tenerse en cuenta para lograr la precisión deseada 
de 20 a 30 nanosegundos [Get93, Hof94, Kra02, Par96].

Debido a que un observador en la superficie terrestre percibe a los satélites en movimiento relativo entre ellos, la Relatividad Especial predice que los relojes atómicos a bordo de los satélites deberían retrasarse debido a la menor velocidad como consecuencia del efecto de dilatación del tiempo de su movimiento relativo [Tay92] .

Por otro lado, los satélites están en órbitas muy por encima de la Tierra, donde la curvatura del espaciotiempo debido a la masa de la Tierra es menor que en la superficie de la Tierra. Una predicción de la relatividad general es que los relojes más cercanos a un objeto masivo funcionen más lentos que los ubicados más lejos. Como tal, cuando se ve desde la superficie de la Tierra, los relojes en los satélites parecen estar funcionando más rápido que los relojes idénticos en la superficie terrestre. Por lo tanto la Relatividad General predice que los relojes en cada satélite GPS deberían adelantarse a los relojes terrestres [Ell88, Cla80].

Por lo tanto, considerando los efectos simultáneos de la Relatividad Especial y General, es decir teniendo presente la velocidad de los satélites y sus alturas orbitales hace que sus relojes marquen más rápido los intervalos de tiempo que cualquier reloj ubicado sobre la superficie terrestre.

\section{Relatividad Especial}

Para calcular la posicicón del dispositivo GPS usaremos la gráfica dada por la figura 1 en la cual se muestran dos sistemas $\vartheta$ y $\vartheta^{\prime}$ con origen común $\vartheta=\vartheta^{\prime}=(0,0)$ en el cual se tienen dos satélites $A$ y $B$ ubicados en los puntos $A=(0,0)$ y $B=\left(L^{\prime}, 0\right)$ ubicado sobre el eje $x^{\prime}$.

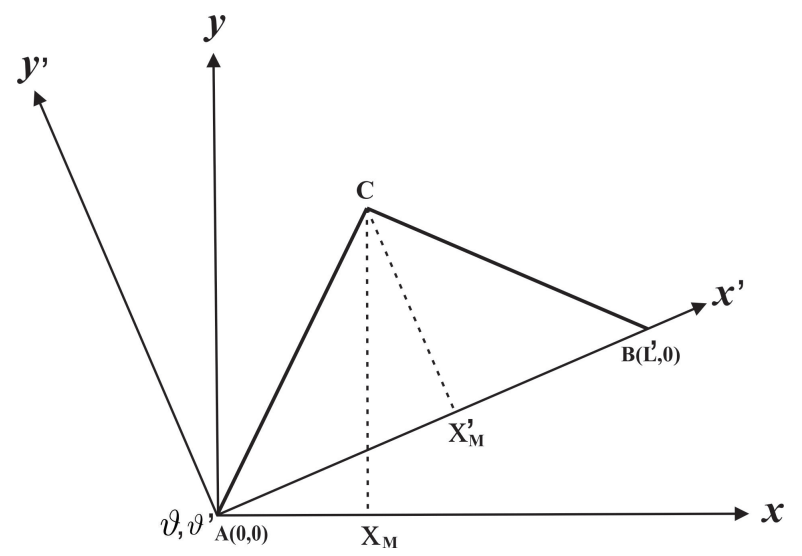

Figura 1: Diagrama espacio-tiempo de los satélites $A$ y $B$.

Usando las transformaciones de Lorentz [Adl75] hallamos las coordenadas de $B$ en el sistema $\vartheta$

$$
x=\frac{L^{\prime}}{\sqrt{1-\beta^{2}}}, \quad t=\frac{L^{\prime} \beta}{\sqrt{1-\beta^{2}}}
$$

donde $\beta=v / c$.

En el sistema de referencia $\vartheta$ intersectando las rectas $\overline{A C}$ y $\overline{B C}$ calculamos las coordenadas del punto $C$

$$
\left(x_{C}\right)_{\vartheta}=\frac{L^{\prime}}{2} \sqrt{\frac{1+\beta}{1-\beta}}, \quad\left(x_{C}\right)_{\vartheta^{\prime}}=\frac{L^{\prime}}{2}
$$

Para calcular la velocidad del satélite usamos la igualdad entre la fuerza gravitacional y la fuerza centrípeta

$$
\frac{G M_{\oplus} m_{s}}{r_{s}^{2}}=\frac{m_{s} v_{s}^{2}}{r_{s}}
$$

cuyos datos de las variables se encuentran en la Tabla 1 [Ahr09]; donde el radio del satélite $\left(r_{s}\right)$ es igual a la suma del radio de la tierra mas la altura a la que se encuentra el mismo $\left(R_{\oplus}+h\right)$.

\begin{tabular}{|l|l|}
\hline Datos & Medidas \\
\hline \hline Masa Tierra $M_{\oplus}$ & $5.9736 \times 10^{24} \mathrm{~kg}$ \\
\hline Radio Tierra $R_{\oplus}$ & $6371000 \mathrm{~m}$ \\
\hline Constante Gravitacional $G$ & $6.672 \times 10^{-11} \mathrm{~m}^{3} / \mathrm{kg} \mathrm{s}^{2}$ \\
\hline Radio satélite $r_{s}$ & $26371000 \mathrm{~m}$ \\
\hline Velocidad de la luz c & $299792458 \mathrm{~m} / \mathrm{s}$ \\
\hline
\end{tabular}

Tabla 1: Valores de constantes que permiten calcular la velocidad del satélite.

Reemplazando los datos adecuados encontramos un valor aproximado de la velocidad del satélite dado por

$$
v_{s}=3.9 \mathrm{~km} / \mathrm{s}
$$

entonces

$$
\beta=\frac{v_{s}}{c}=1.3 \times 10^{-5}
$$

Por otro lado, la distancia aproximada entre satélites, para una red de 24 satélites viene dada por

$$
L^{\prime}=\frac{2 \pi r_{s}}{24}=6903.911 \mathrm{~km}
$$

Calculando las coordenadas de $C$ se tiene

$$
\begin{gathered}
\left(x_{C}\right)_{\vartheta}=3452 \mathrm{~km} \\
\left(x_{C}\right)_{\vartheta^{\prime}}=3451.956 \mathrm{~km}
\end{gathered}
$$

Calculando el desfase de $x_{C}$ respecto a los dos sitemas de referencia $\vartheta$ y $\vartheta^{\prime}$ tenemos

$$
\triangle\left(x_{C}\right)=3452-3451.956=0.0445 \mathrm{~km}=44.5 \mathrm{~m}
$$

Este desfase de $44.5 \mathrm{~m}$, en la localización del punto $C$, sucede al no considerar la Relatividad Especial.

Por otro lado debemos tener presente que para el sistema $\vartheta^{\prime}$ las señales de los satélites es simultánea pero no lo es para el sistema $\vartheta$. Considerando que el satélite $A$ envía la señal en $\left(t_{A}\right)_{\vartheta}=0$ el satélite $B$ enviaría la señal en un tiempo dado por

$$
\left(t_{B}\right)_{\vartheta}=\frac{1}{c} \frac{L^{\prime} \beta}{\sqrt{1-\beta^{2}}}=3 \times 10^{-7}
$$


Ahora calculamos los tiempos correspondientes al satélite $\left(t_{s}\right)$ y al del dispositivo GPS $\left(t_{o}\right)$ usando las coordenadas de Lorentz [Rin91, Gou13]

$$
t_{o}=\frac{t_{s}}{\sqrt{1-\beta^{2}}}
$$

mediante la expansión de Taylor realizamos la siguiente aproximación

$$
\begin{array}{r}
\frac{t_{o}}{t_{s}}=\frac{1}{\sqrt{1-\beta^{2}}} \approx\left(1-\frac{1}{2}\left(\frac{v_{s}}{c}\right)^{2}\right)= \\
1-0.8461703676 \times 10^{-10}
\end{array}
$$

Notamos que entre el tiempo del satélite y del dispositivo GPS existe una diferencia de $0.8461703676 \times 10^{-10} \mathrm{~s}$.

\section{Relatividad General}

Una solución a las ecuaciones de campo de Einstein apropiada para relacionar la Teoría de la Relatividad General con el GPS es la solución de Schwarzschild, dada por [Wei72, Mis71]:

$$
\begin{array}{r}
d s^{2}=\left(1-\frac{2 m}{r}\right) d t^{2}-\left(1-\frac{2 m}{r}\right)^{-1} d r^{2} \\
-r^{2}\left(d \theta^{2}+\operatorname{sen}^{2} \theta d \phi^{2}\right)
\end{array}
$$

Esta expresión corresponde a una solución: estática, en el vacío y esféricamente simétrica.

La métrica de Schwarzschild nos permite relacionar el tiempo propio de dos partículas a distintas alturas; sea $t_{o}$ el tiempo propio en la superficie de la tierra y $t_{s}$ el tiempo propio a una altura $h$ respecto de la superficie terrestre.

Estos dos tiempos se relacionan por:

$$
t_{o}=t_{s}\left(\frac{1-\frac{2 M_{\oplus}}{R_{\oplus}}}{1-\frac{2 M_{\oplus}}{r_{s}}}\right)^{1 / 2}
$$

Usando la aproximación mediante la expansión de Taylor se tiene

$$
\frac{T_{o}}{T_{s}}=1-\frac{M_{\oplus}}{R_{\oplus}}+\frac{M_{\oplus}}{r_{s}}
$$

usando el factor de conversión $G / c^{2}$ para convertir la masa al sistema internacional, encontramos que

$$
\begin{aligned}
\frac{M_{\oplus}}{R_{\oplus}} & =-\frac{G M_{\oplus}}{c^{2} R_{\oplus}}=-6.960543709 \times 10^{-10} \\
\frac{M_{\oplus}}{r_{s}} & =-\frac{G M_{\oplus}}{c^{2} r_{s}}=-1.681605702 \times 10^{-10}
\end{aligned}
$$

entonces

$$
\frac{T_{o}}{T_{s}}=1+5.278938007 \times 10^{-10}
$$

Por tanto, el tiempo propio entre un dispositivo GPS y un satélite, ambos estáticos, presentan una diferencia de $5.278938007 \times 10^{-10} \mathrm{~s}$.

\section{Relatividad Especial más Relatividad Ge- neral}

De acuerdo a los resultados anteriores, se ha notado que el desfase de tiempo se presenta usando tanto la Relatividad Especial como la Relatividad General.

Seguidamente calculamos el desfase de tiempos entre el dispositivo GPS y el satélite aplicando simultaneamente las correciones debidas a las ecuaciones de la Relatividad Especial y General. De las expresiones (12) y (18) se tiene:

$$
\begin{aligned}
\frac{T_{o}}{T_{s}}=5.278938007 \times 10^{-10}- & 0.8461703676 \times 10^{-10} \\
& =4.432767639 \times 10^{-10}
\end{aligned}
$$

Esto significa que por cada segundo, respecto a un observador en un marco de referencia en Tierra, el satélite tiene un desfase de $4.432767639 \times 10^{-10} \mathrm{~s}$ por lo tanto en un día, respecto a un observador, en la superficie terrestre, el satélite habrá acumulado un desfase de $\triangle t=3.82991124 \times 10^{-5} \mathrm{~s}$. Con este valor se puede calcular el desfase espacial en un día, resultando:

$$
d=c \times \triangle t=11481.78505 \mathrm{~m}=11.48178505 \mathrm{~km}
$$

Si no se toma en cuenta las correcciones relativistas el Sistema de Posicionamiento Global marcará, en un día, aproximadamente $11.5 \mathrm{~km}$ alejados del dispositivo GPS, lo cual haría inservible el sistema GPS.

\section{Conclusiones}

La Relatividad Especial y la Relatividad General son herramientas fundamentales para obtener la precisión requerida por el GPS. Si dichos efectos no se tiene en cuenta adecuadamente, se producirán errores inaceptablemente grandes en la navegación GPS y la transferencia de tiempo.

La Relatividad Especial produce un desfase de tiempo de $0.8461703676 \times 10^{-10} \mathrm{~s}$, mientras que la Relatividad General $5.278938007 \times 10^{-10} \mathrm{~s}$, notamos que la Relatividad General presenta mayor desfase temporal que la Relatividad especial, por tanto contribuye con un mayor índice de correción en la calibración de los relojes.

La contribución de las dos relatividades es importante ya que en un día el satélite habrá acumulado un desfase temporal de $3.82991124 \times 10^{-5} \mathrm{~s}$ el cual se traduce en un desfase espacial de aproximadamente $11.5 \mathrm{~km}$. 


\section{Referencias}

[Adl75] Adler, R., Bazin, M. and Schiffer, M. (1975). Introduction to General Relativity. New York: McGraw Hill.

[Ahr09] Ahrens, T. (2009). Global Earth Physics. A Handbook of Physical Constants, American Geophysical Union, Washington, D.C.

[Cla80] Clarke, C. (1980). Elementary General Relativity. Halsted Press.

[Ell88] Ellis, G. and Williams, R. (1988). Flat and Curved Space-Times. Oxford University Press.

[Elr02] El-Rabbany, A. (2002). Introduction to GPS: The Global Positioning System. Artech House Mobile Communications Series

[Get93] Getting, I. (1993). The Global Positioning System. IEEE Spectrum, Dec-1993.

[Gou13] Gourgoulhon, E. (2013). Special Relativity in General Frames, From Particles to Astrophysics, Springer.
[Hof94] Hoffman-Wellenhoff, B., Lichtenegger, H. and Collins, J. (1994). Global Positioning. 3rd Ed. New York: Springer-Verlag Wien.

[Kra02] Krasner, N., Marshall, G. and Riley, W. (2002). Position Determination Using Hybrid GPS/Cellphone Ranging. Proceedings of the 15th International Technical Meeting of the Satellite Division of The Institute of Navigation, Portland.

[Mis71] Misner, C., Thorne, K. and Wheeler, J. (1971). Gravitation. San Francisco: W. H. Freeman \& Co.

[Par96] Parkinson, B. (1996). Global Positioning System: Theory and Applications, Chap. 1: Introduction and Heritage of NAVSTAR, the Global Positioning System. pp. 3-28, American Institute of Aeronautics and Astronautics, Washington, D.C.

[Rin91] Rindler, W. (1991). Introduction to Special Relativity. Oxford University Press.

[Tay92] Taylor, E. and Wheeler, J. (1992). Spacetime Physics: Introduction to Special Relativity. New York: 2nd ed. W. H. Freeman \& Company.

[Wei72] Weinberg, S. (1972) Gravitation and Cosmology. New York: John Wiley \& Sons. 